\title{
Probing the Structure and Dynamics of Confined Water in AOT Reverse Micelles
}

\author{
Anna Victoria Martinez, Laura Dominguez, Edyta Małolepsza, Adam Moser $\stackrel{\ddagger}{\ddagger}$ Zack Ziegler, \\ and John E. Straub* \\ Department of Chemistry, Boston University, Boston, Massachusetts 02215, United States
}

\section{Supporting Information}

ABSTRACT: Reverse micelles are attractive nanoscale systems used for the confinement of molecules in studies of structure and chemical reactions, including protein folding, and aggregation. The simulation of reverse micelles, in which a water "pool" is separated from a nonpolar bulk phase by a surfactant layer, poses significant challenges to empirical force fields due to the diversity of interactions between nonpolar, polar, and charged groups. We have explored the dependence of system density, reverse micelle structure, and water configurational relaxation times as a function of reverse micelle composition, including water:surfactant ratio, absolute number of water molecules, and force field using molecular dynamics simulations. The resulting structures and dynamics are found to depend more on the force field used than on varying interpretations of the water:surfactant ratio in terms of absolute size of the reverse micelle. Substantial deviations from spherical reverse micelle geometries are observed in all unrestrained simulations. Rotational anisotropy decay times and water residence times show a strong dependence on force field and water model used, but power-law relaxation in time is observed independent of the force field. Our results suggest the need for further experimental study of reverse micelles that can provide insight into the distribution and dynamics of shape fluctuations in these complex systems.

\section{INTRODUCTION}

Reverse micelles (RMs) have been the subject of experimental studies since the early 1940s. ${ }^{1}$ Reverse micelles allow for a tunable amount of water to be encapsulated within a membrane like environment. The water cores of RMs have been found to behave similarly to cavities found in biological systems, ${ }^{2}$ where the water-surfactant interface of reverse micelles mimics that behavior in a less complex environment and on a smaller scale. ${ }^{3}$ These properties make RMs powerful tools in which to probe confinement effects and dehydration on biological molecules. $^{4-6}$

Sodium bis(2-ethylhexyl) sulfosuccinate (AOT) is a commonly used surfactant as it readily forms RMs in nonpolar solvents without a cosurfactant. AOT's branched structure allows the molecules to pack together and dissolve large amounts of water. ${ }^{7,8}$ The shape, size, and composition of RMs have been studied using many experimental techniques including $\mathrm{NMR},{ }^{9}$ small-angle X-ray scattering (SAXS), ${ }^{10}$ dynamic light scattering (DLS), ${ }^{11}$ and pulsed gradient stimulated echo. ${ }^{12}$ The size of reverse micelles depends in part on their water loading $\left(w_{0}\right)$, which is the ratio of water molecules to surfactant molecules: ${ }^{13}$

$$
w_{0}=\frac{\left[\mathrm{H}_{2} \mathrm{O}\right]}{[\text { surfactant }]}
$$

The absolute composition of reverse micelles is still a matter of debate. The two most used estimates come from Amararene et al. ${ }^{10}$ using SAXS and volumetric measurements and Eicke et al. ${ }^{11}$ using analytic ultracentrifuge. These estimates disagree by as much as $35 \%$ for low water loadings $\left(w_{0}=3-10\right)$, and the Waks work reports very small molecular water volumes for these. Further, the shape of AOT RMs is also still in question. ${ }^{14}$

Significant computational work has been performed using AOT RMs. ${ }^{15-25}$ Some earlier simulations employed implicit solvent models in which the surfactant head groups were represented by a single charge interaction site, the water was treated explicitly, and a continuum potential was used to represent the nonpolar solvent and the surfactant tail groups. ${ }^{15-17}$ While useful, the implicit solvent model employed in that pioneering work failed to provide structural information for the surfactant or the interaction between the surfactant and the nonpolar solvent. These simulations also spherically constrained the water core of the RM, not taking into account any deformation in shape or water penetration into the surfactant region of the RM.

To obtain more detailed structural information about reverse micelles, Abel et al. ran the first all atom simulations of AOT

Received: March 5, 2013

Revised: $\quad$ May 16, 2013

Published: May 20, 2013 
RMs of $w_{0}=3,5$, and 7 in isooctane. They observed that within a few hundred picoseconds the RMs deviated from their original spherical shape and became elliptical. The properties of water also became more bulk-like as the RMs increased in size. This work led to the first insights into the nature of the equilibrium structural ensemble of AOT RMs. ${ }^{18}$

Brodskaya et al. developed a coarse-grained model for AOT surfactant in hexane. They simulated RMs of $w_{0}=2,3,4$, and 5 and studied the effects of size and water content on structure. ${ }^{19}$ In their 2-ns simulations the RMs, which were initially spherical, evolved into elliptical shapes. The change in shape was less pronounced as the RMs increased in size. ${ }^{20}$ They also studied the translational diffusion within the reverse micelles and found that mobility of ions, polar heads of AOT, and water increased as the RMs increased in size. ${ }^{21}$ Using a united atom model Chowdhary and Ladanyi simulated AOT RMs $\left(w_{0}=2-\right.$ 7.5) in isooctane and examined the effect of RM size on structure. $^{22}$

Tian and Garcia performed landmark simulations of the selfassembly of $\mathrm{RMs}^{23}$ for compositions relevant to $w_{0}=6$ and 11 . The simulated dynamics were extensive, showing the formation of small RMs and RM fusion ultimately forming RMs of an elliptical structure.

Graeve et al. performed experimental and computational studies (including self-assembly) investigating the size and shape of AOT RMs. In their initial work they observed significant deviations from the initial spherical shape for small RMs within the $1 \mathrm{~ns}$ of simulation time. They also noted that there is less fluctuation in shape for the larger RMs $\left(w_{0}>10\right){ }^{24}$ In a subsequent extensive and critical study of RM structure, they compared the results of their computational work to dynamic light scattering experiments on the same systems. They observed highly qualitative agreement between their simulations and experiment, and found nonspherical shapes for the reverse micelles. ${ }^{25}$

Because previous theoretical work has generally employed spherical restraints, focused on a single $w_{0}$, used a single absolute composition estimate, or used a single force field, this work broadens the understanding of how these choices affect AOT RM simulations. In this work we used two force fields to simulate 12 reverse micelle systems (four spherically restrained, eight unrestrained) of $w_{0}=6$ and 10 using both experimental composition estimates. The compositions of the reverse micelles systems are outlined in Table 1 .

\section{METHODS}

RM systems were generated using the CHARMM32 package with the CHARMM27 all atom force field for proteins and

Table 1. Reverse Micelle Compositions Including the Water Loading $\left(w_{0}\right)$, Total Number of AOT and Water Molecules $\left(n_{\mathrm{AOT}}\right.$ and $\left.n_{\mathrm{H} 2 \mathrm{O}}\right)$, the Mole Fraction of Isooctane $\left(\chi_{\mathrm{ISO}}\right)$, and the Radius of the Water Pool for Each System ${ }^{a}$

$\begin{array}{crrrrc}\text { composition } & w_{0} & n_{\mathrm{AOT}} & n_{\mathrm{H}_{2} \mathrm{O}} & \chi_{\mathrm{ISO}} & \text { water radius }(\AA) \\ \text { Eicke } & 6 & 50 & 300 & 0.81 & 12.00-16.00 \\ \text { Eicke } & 10 & 97 & 970 & 0.80 & 18.00-22.00 \\ \text { Waks } & 6 & 76 & 456 & 0.81 & 11.25-15.25 \\ \text { Waks } & 10 & 129 & 1290 & 0.83 & 18.00-22.00\end{array}$

${ }^{a}$ The values given for the radii were the initial values for the unrestrained systems and the distance paramters placed on the spherically restrained systems throughout the simulations. lipids and the TIP3P water model for CHARMM. ${ }^{26}$ CHARMM parameters for AOT and isooctane were taken from the work of Abel et al. ${ }^{18}$ For the spherically restrained RMs a massless dummy atom was fixed in the center of the RM. A harmonic restraint of $2 \mathrm{kcal} / \mathrm{mol} / \AA$ was placed on the sulfur atom of each AOT molecule to keep it within a certain distance of the dummy atom (see Table 1). The distance restraints were chosen to agree with experimental measurements of the solution density in refs 10 and 11 .

The starting structures for the unrestrained RMs were used for the GROMOS systems which were generated with the GROMOS96 53a6 united atom force field. ${ }^{27}$ As there is no previous work in the literature modeling AOT with this force field, we attempted to remain as "force field consistent" as possible in using GROMOS to generate the AOT headgroup parameters. The parameters for the sulfur headgroup began with a choice of the sulfur parameters used for DMSO.

$\mathrm{NAMD}^{28}$ and GROMACS were used for the production runs of the CHARMM and GROMOS systems, respectively. The cutoff for the short-range electrostatics calculations was set to be $12 \AA$, and Ewald was used for the long-range electrostatics. The temperature was held constant at $300 \mathrm{~K}$, and the pressure was held constant at 1 atm using the Langevin ${ }^{29,30}$ (for NAMD) and Berendsen ${ }^{31}$ (for GROMACS) pistons. There are alternatives based on extended-Lagrangian methods, such as a combination of Nose-Hoover chains with an Anderson piston, that are more elegant. However, we have not observed thermostat-dependent differences in the results for dynamics or equilibrium averaging for systems of the size studied in this work. SHAKE was used to keep bonds containing hydrogen atoms rigid. Each trajectory was run for $25 \mathrm{~ns}$ saving data every 0.1 ps. Analysis of all systems was performed using CHARMM, GROMACS, and MDAnalysis. ${ }^{32}$

\section{RESULTS}

Agreement between Simulated and Observed Densities. Experimentally, the density of the $w_{0}=6 \mathrm{RMs}$ is observed to be higher than the density of the $w_{0}=10 \mathrm{RMs}^{10}$ The values we report for the unrestrained CHARMM RMs in Figure 1 follow this trend. For the density calculations we used CHARMM to calculate the volume and density of a water box to determine appropriate values for probe radius and grid spacing, which were then used to calculate the volumes and densities for the reverse micelle systems. In all cases, the Eicke composition leads to a lower density than the Waks composition. While our simulated densities may be higher or lower than experimental values, all are within approximately $5 \%$ of these values, regardless of composition or force field (for GROMOS data see Supporting Information). The densities of the spherically restrained RMs are higher than for the unrestrained RMs, but are still within $2 \%$ of the experimental values.

Unrestrained RMs are Not Spherical. The initial geometry of all simulated reverse micelles was spherical. In contrast to the shape of the spherically restrained RMs, the shape of the unrestrained RMs changed significantly. The unrestrained CHARMM RMs evolved from spheres to rod-like shapes, and the unrestrained GROMOS RMs became disc- and donut-like. To quantify the changes in geometry, we calculated several shape parameters including the moments of inertia, the semiaxes, and the eccentricity of each RM. The moments of inertia $I_{1}, I_{2}, I_{3}$ for an ellipse with semiaxes $a, b, c$ are 


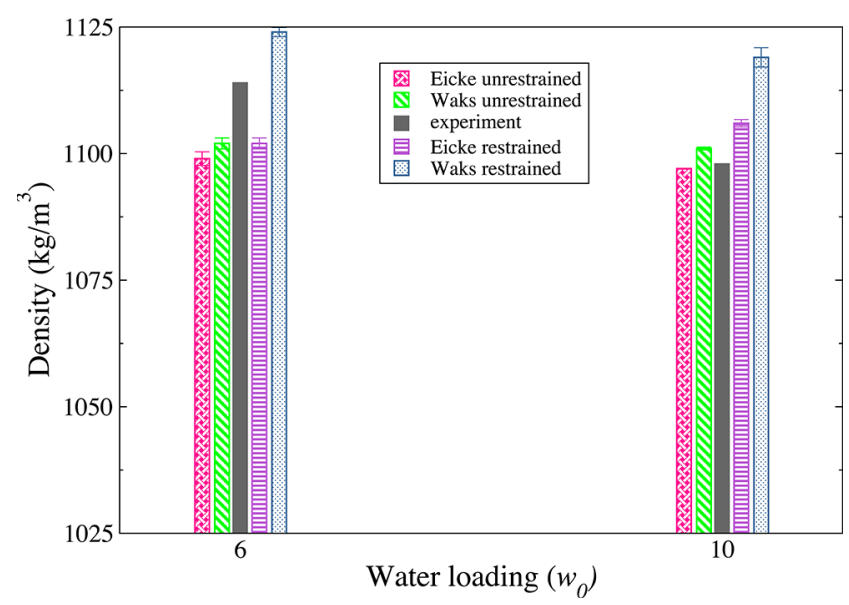

Figure 1. Densities $\left(\mathrm{kg} / \mathrm{m}^{3}\right)$ for all simulated CHARMM RMs. The experimental values are in dark gray. The densities for the unrestrained RMs are to the left of the experimental values, and the densities for the restrained RMs are to the right. All calculated values for the CHARMM simulations are within $2 \%$ of the reported experimental densities.

$$
\begin{aligned}
& I_{1}=\frac{m}{5}\left(b^{2}+c^{2}\right) \\
& I_{2}=\frac{m}{5}\left(c^{2}+a^{2}\right) \\
& I_{3}=\frac{m}{5}\left(a^{2}+b^{2}\right)
\end{aligned}
$$

For spherical objects, $I_{1} \approx I_{2} \approx I_{3}$ and $a \approx b \approx c$. For a prolate ellipsoid (or a rod), $I_{1} \approx I_{2}>I_{3}$, and $a \approx b<c$. For an oblate ellipsoid (or a disc) $I_{1} \approx I_{2}<I_{3}$, and $a \approx b>c$. The eccentricity, $e$, of a shape is given by

$$
e=\sqrt{1-\frac{c^{2}}{a^{2}}}
$$

where $e$ is zero for a perfect sphere and $e \rightarrow 1$ for disc- and rodlike shapes. ${ }^{18}$

Table 2 shows the average moments of inertia, the semiaxes, and the eccentricity for each of the CHARMM unrestrained RMs for the last $15 \mathrm{~ns}$ of simulation. The differences in the values of $I_{1}, I_{2}$, and $I_{3}$ and $a, b$, and $c$ indicate nonspherical shapes. The moments of inertia for the CHARMM systems fluctuated significantly tending toward two large moments and one smaller one. The semiaxes also fluctuated, tending toward two short semiaxes and one long semiaxis, indicating a rod-like shape for these systems. The moments of inertia and the semiaxes for the GROMOS systems fluctuated generally indicating that the RMs shifted from spheres to disc-like shapes.
The eccentricity for all systems increased with time representing the shape evolution away from spherical. Figure 2 shows the eccentricity parameter for the CHARMM

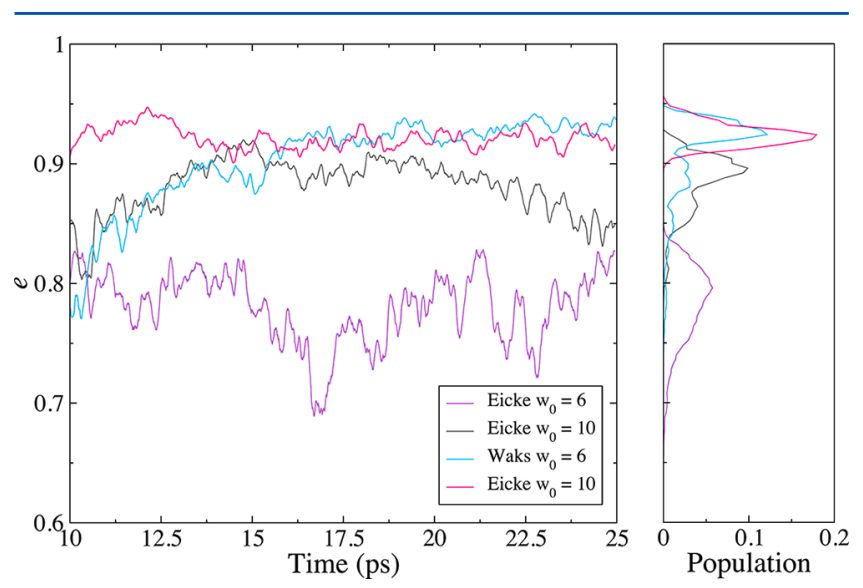

Figure 2. Eccentricity parameter for the CHARMM unrestrained RMs for the last $15 \mathrm{~ns}$ of simulation. The eccentricity parameter is plotted versus time (left) with the normalized histogram of eccentricity values shown for the last $15 \mathrm{~ns}$ (right).

unrestrained RMs from 10 to $25 \mathrm{~ns}$ along with the distribution of eccentricity values for each RM. For the restrained RMs, there was little fluctuation for all values (data not shown). Figure 3 shows images of the unrestrained RMs for the CHARMM force field at 0,15 , and 25 ns following initial "equilibration." GROMOS data are available in the Supporting Information.

On the basis of these results, we conclude that the shape of the RMs is more dependent on force field than composition. One main difference between the two force fields is the partial charges on the atoms. The CHARMM force field assigns a nonzero partial charge to every atom. ${ }^{26}$ The AOT and isooctane parameters of Abel et al. follow this pattern. ${ }^{18}$ In the GROMOS force field not every atom has a nonzero partial charge. ${ }^{27}$ For AOT molecules the only atoms with nonzero partial charges are the sulfur, the oxygens, and the ester carbons. The rest of the aliphatic carbons have partial charges of zero. Because of the lack of partial charges in the GROMOS parameters there are no Coulombic interactions between the water molecules and the AOT tails, which apparently leads to a stabilization in the geometry of the reverse micelles. In the CHARMM systems the waters interact more strongly with the AOT head groups and hydrate the aliphatic tails to some extent. Water observed to penetrate the surfactant layer allows the shape of the RMs to be more flexible.

Water Relaxation Shows Highly Nonexponential Behavior. The rotational anisotropy correlation function can

\begin{tabular}{|c|c|c|c|c|c|c|c|c|}
\hline composition & $w_{0}$ & $I_{1}$ & $I_{2}$ & $I_{3}$ & $a$ & $b$ & $c$ & $e$ \\
\hline \multicolumn{9}{|c|}{ Unrestrained RMs-CHARMM force field } \\
\hline Eicke & 6 & $4.1 \pm 0.2$ & $5.1 \pm 0.3$ & $6.3 \pm 0.2$ & $25.8 \pm 1.1$ & $21.9 \pm 0.9$ & $16.1 \pm 0.5$ & $0.78 \pm 0.03$ \\
\hline Waks & 6 & $6.4 \pm 0.8$ & $17.1 \pm 2.0$ & $18.4 \pm 1.9$ & $47.4 \pm 2.3$ & $18.6 \pm 0.8$ & $16.9 \pm 0.6$ & $0.93 \pm 0.01$ \\
\hline Eicke & 10 & $12.2 \pm 1.0$ & $30.3 \pm 2.9$ & $31.7 \pm 2.4$ & $45.2 \pm 2.8$ & $23.6 \pm 1.3$ & $21.1 \pm 0.9$ & $0.88 \pm 0.02$ \\
\hline Waks & 10 & $19.8 \pm 1.0$ & $55.8 \pm 3.2$ & $61.0 \pm 2.3$ & $54.8 \pm 1.6$ & $27.8 \pm 1.6$ & $21.2 \pm 1.2$ & $0.92 \pm 0.01$ \\
\hline
\end{tabular}
be calculated by taking the dot product of the unit vectors, $\hat{u}$, in

Table 2. Average Values for Last $15 \mathrm{~ns}$ of Simulation Time for the Moments of Inertia $I_{1}, I_{2}, I_{3}\left(10^{6}\right.$ amu* $\left.\AA^{2}\right)$, Semiaxes $a, b, c$ $\left(\AA^{2}\right)$, and Eccentricity $e$ for CHARMM Unrestrained RM Systems 


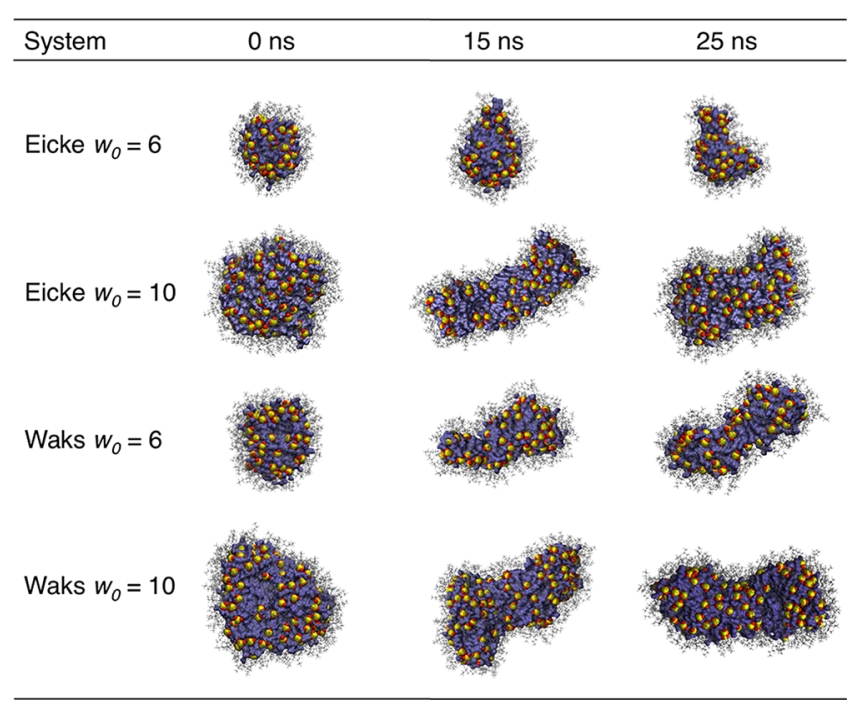

Figure 3. Structures of the unrestrained reverse micelles for the CHARMM force field. The images from left to right show the structures of the RMs at 0,15 , and $25 \mathrm{~ns}$. The AOT sulfur head groups are represented by yellow sulfur atoms and red oxygen atoms. The AOT tails are in gray, and the water is in blue.

the direction of the $\mathrm{O}-\mathrm{H}$ bonds, and then calculating the correlation function of the second Legendre polynomial, $P_{2}$, as

$$
C_{2}(t)=\left\langle P_{2}(\hat{u}(t) \cdot \hat{u}(0))\right\rangle
$$

where $P_{2}(x)=\left(3 x^{2}-1\right) / 2$ and $x=\cos \theta=(\hat{u}(t) \cdot \hat{u}(0)) \cdot{ }^{33}$ The second Legendre polynomial is relevant as it is used in analyzing order of liquid crystals. ${ }^{34}$ The limit of $\left\langle P_{2}(x)\right\rangle \rightarrow 0$ indicates a random, isotropic system with fast decay times and $\left\langle P_{2}(x)\right\rangle \rightarrow 1$ indicates a more ordered system with slow decay times. $^{34}$

The second-rank rotational anisotropy decay autocorrelation function (ACF) of the water was computed and compared to experimental values. ${ }^{35}$ The rotational anisotropy decay ACF provides insight on restrictions in structural dynamics of water in confined areas, such as RMs of $w_{0}<20$. Experiments show that confined water rotates slowly and has a long rotational anisotropy decay time. ${ }^{36}$ Rotational anisotropy decay times for water in reverse micelles may be extracted by fitting the ACFs to exponential functions. ${ }^{35-37}$ The rotational anisotropy decay of bulk water and water in large RMs $\left(w_{0}=20,40,60\right)$ can be fit to a single exponential function. The rotational anisotropy decay of water in small RMs $\left(w_{0}<20\right)$ is not observed to decay as a single exponential, with confined water exhibiting multiple $^{38}$ or stretched exponential ${ }^{35}$ behavior and multiple relaxation times on short time scales $(\sim 10-20 \mathrm{ps})$, and power law decay on longer time scales. ${ }^{39-41}$ Piletic et al. found that for RMs of $w_{0} \leq 10$, the water orientational relaxation behaved biexponentially with long decay times of 50, 30, and 18 ps and short decay times of $0.9,1.0$, and 1.5 ps for RMs of $w_{0}=2,5$, and 10 , respectively. ${ }^{36}$

We calculated the rotational anisotropy autocorrelation functions for the last 2000 ps of our simulations averaging over 1000 ps windows. For short time periods ( 20 ps), the water rotational anisotropy relaxation in our simulations can be described either by using a sum of three exponential functions $^{38,42}$ or a stretched exponential function. ${ }^{40,41,43}$ While the stretched exponential function does provide a good fit to short time data, an intuitively appealing approach employs a sum of exponentials where "types of water" are identified in the system with each "type" being associated with a particular mean relaxation time. Examples include studies of reverse micelles $^{38}$ and studies of water at the lipid-water interface ${ }^{42}$ that suggest the water dynamics are best described by a sum of three exponentials. Biswas et al. proposed a triexponential model in which the rotational anisotropy decay times were calculated for three layers of water-a surface layer, an intermediate layer, and a central layer. Each layer exhibited triexponential behavior, with the surface layer having the slowest decay times and the intermediate layer having the fastest decay times. ${ }^{38}$

Figure 4 shows the rotational anisotropy ACFs with triexponential fits (top) and stretched exponential fits (bottom)
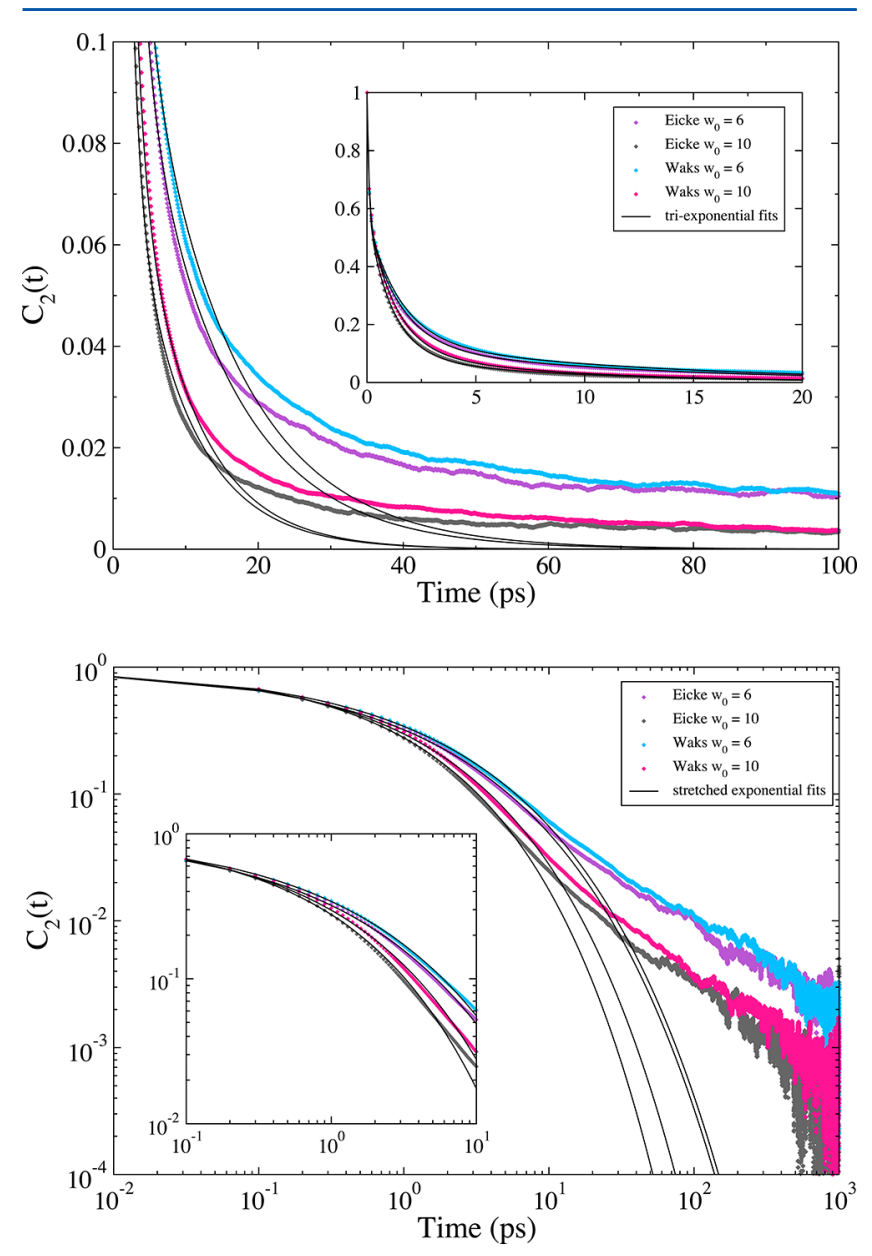

Figure 4. Rotational anisotropy decay autocorrelation functions for the unrestrained CHARMM reverse micelles. The inset plots show that the autocorrelation functions fit well to a triexponential function (top) or a stretched exponential function (bottom) up to approximately 10 or 20 ps. The large plots show that these fits fail to describe the correlation functions on longer time scales.

for the unrestrained CHARMM RMs. The plots show that the ACFs fit well to triexponential $\left(C_{0} \mathrm{e}^{-x / \tau}{ }_{0}+C_{1} \mathrm{e}^{-x / \tau_{1}}+C_{2} \mathrm{e}^{-x / \tau_{2}}\right)$ or stretched exponential $\left(\mathrm{e}^{-(x / \tau)^{\beta}}\right)$ functions up to $\sim 20 \mathrm{ps}$, but these functions fail to capture the longer decay time features of the ACF. For the stretched exponential fits we obtained $\tau$ values ranging from 0.61 to $0.85 \mathrm{ps}$ and $\beta$ values between 0.42 and 0.5 for the restrained and unrestrained CHARMM RMs. Pieniazek et al. ${ }^{35}$ obtained $\beta$ values ranging from 0.17 to 0.37 , 
which is indicative of collective behavior for the water. The $\beta$ values they report are for RMs of $w_{0}=2,4$, and 7.5. In smaller RMs, water dynamics will be more collective which may explain the difference in our values. While the GROMOS rotational anisotropy decay times are much slower, triexponential functions also fit the first 20 ps of the ACFs, but fail to describe the longer decay times. Further analysis is provided in the Supporting Information.

For longer time periods of up to approximately 1000 ps, the stretched exponential model fails to capture the water dynamics. Data from our CHARMM simulations are better described by a pair of power laws of the form $t^{-b_{1}}$ and $t^{-b_{2}}$ where the $b_{1}$ component fits longer decay times and the $b_{2}$ component fits shorter decay times. In Figure 5 the rotational anisotropy

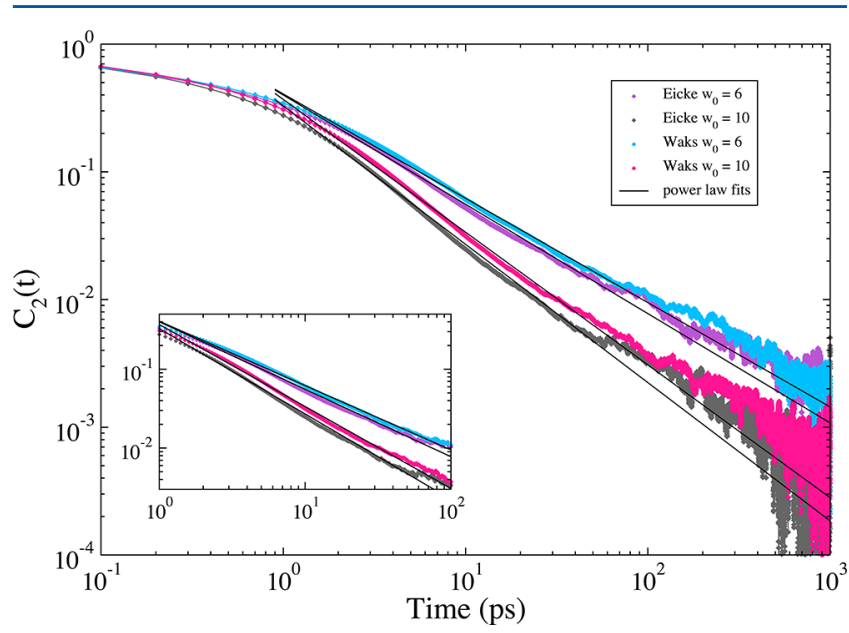

Figure 5. Rotational anisotropy decay autocorrelation functions for the unrestrained CHARMM reverse micelles on a log-log scale. The plot shows that the autocorrelation functions are described well with a power law decay from 1 to $100 \mathrm{ps}$.

ACFs along with the power law fits are plotted on a log-log plot. The figure shows that the ACFs fit well to a power law $t^{-b_{1}}$ after $\sim 1$ ps with $b_{1}$ values between 0.8 and 1.1. A second power law $t^{-b_{2}}$ (not shown on graph) describes the short times up to 1 ps with $b_{2}$ values between 0.33 and 0.4 . As the reverse micelles increase in size, the behavior of the water approaches that of bulk.

Several other groups have analyzed the rotational anisotropy decay of water in confined environments other than reverse micelles. ${ }^{39-41,43-45}$ They find that while the initial decay (up to 10 or 20 ps) can be characterized well with a stretched exponential or sum of exponential functions, the longer decay times are described more accurately by a power law or a pair of power laws.

Our observation of power-law relaxation of the rotational anisotropy decay for water in reverse micelles is consistent with observations by Laage and Thompson in their computational work on water confined in silica pores, ${ }^{39}$ and experimental work by Scodinu et al. ${ }^{44}$ and Farrer et al. ${ }^{45}$ using optical Kerr effect spectroscopy to study orientational dynamics of confined water.

In addition to the rotational anisotropy decay, we calculated the residence times of the water molecules with respect to each AOT in the unrestrained RMs for the last 2000 ps. We defined residence time to be the length of time an individual water molecule remained within $4 \AA$ of an AOT headgroup oxygen. The distributions of the residence times for the unrestrained CHARMM and GROMOS systems are shown on a log-log plot in Figure 6. The residence time distributions, like the rotational anisotropy, decay as power laws. The CHARMM

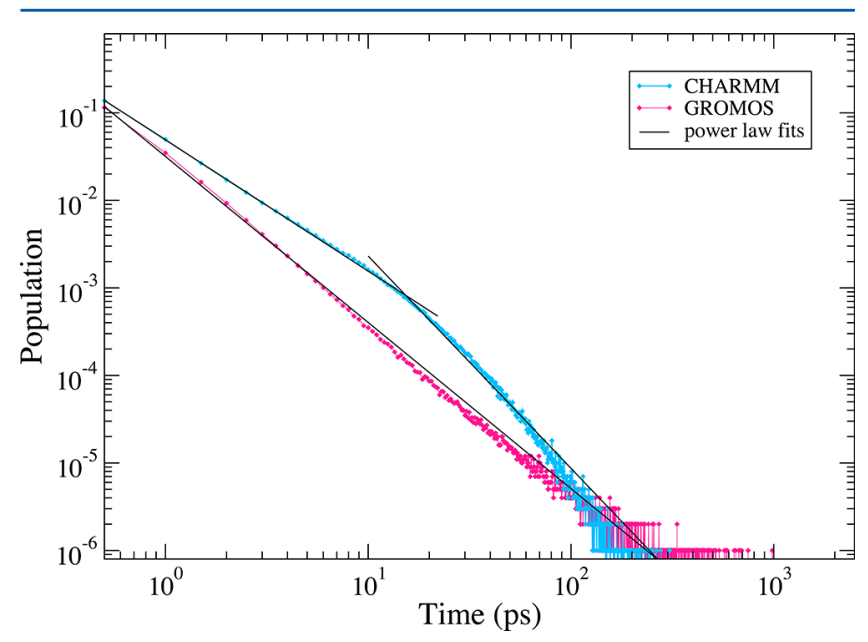

Figure 6. Distribution of water residence time for all AOTs head groups in the unrestrained RMs.

data is fit well by two power laws and the GROMOS to one. This graph shows that in general the TIP3P water has shorter residence times than SPC water. The GROMOS simulations have a larger fraction of waters with residence times of 100 ps or longer which may contribute to the long rotational anisotropy decay times.

\section{DISCUSSION}

Previous RM simulations by Ladanyi et al., ${ }^{22}$ which used the Eicke composition, resulted in more spherical RMs than what we present here. We believe this difference results from the force field used. It is important to note that each of our simulations was performed using parameters specifically for each force field used. In Ladanyi's model, parameters from more than one force field were used, ${ }^{22}$ which may be partially responsible for the observed differences. ${ }^{46}$ The AOT parameters are similar to those in the GROMOS force field, with nonzero partial charges on the oxygens and ester carbons, but partial charges of zero on the sulfur atom and the remaining aliphatic carbons. The isooctane carbons also have partial charges of zero. This means there are only electrostatic and Coulombic interactions between the water and the AOT headgroup region. This appears to lead to the lack of water penetration to the AOT tails with the majority of the waters remaining in the core of the RM. Two additional simulations of the Waks $w_{0}=6 \mathrm{RM}$ were performed using CHARMM and GROMOS. In the CHARMM simulations we changed the partial charges of the AOT and the isooctane to be the same as those used in Ladanyi's simulations and in the GROMOS simulations we changed the water from SPC to SPC/E. These changes had no significant effects on the shapes of the RMs.

Simulations by Graeve et al. ${ }^{24,25}$ resulted in reverse micelles with cylindrical or elongated donut-like shapes. The type and range of shape fluctuations in our simulations are consistent with those of Graeve and co-workers, which was also consistent with the results of their experimental light scattering studies. ${ }^{25}$

Their work employed the same force field and parameters for AOT as in the CHARMM simulations presented in this work. They studied RMs of various $w_{0}$ by keeping the number of AOT molecules constant and varying the amount of water 
present. They note that the absolute composition of the RMs is less important than the water:surfactant ratio as their simulation results are in good agreement with their experimental studies of the same systems indicating nonspherical RMs.

\section{CONCLUSION}

Reverse micelles are widely used in experimental studies of molecular structure and dynamics, including protein folding and aggregation, using NMR and optical spectroscopies. ${ }^{9-11}$ Owing to the complexity of interactions between the sequestered molecules and the surrounding water pool and surfactant, simulation studies are essential to the complete interpretation of experimental spectroscopic studies of the structure. We have used two force fields to simulate reverse micelles based on two alternative interpretations of the water loading in terms of the absolute numbers of water and surfactant molecules determined by two experimental groups. ${ }^{10,11} \mathrm{We}$ find the properties of the RMs to be more dependent on the size ( $w_{0}=6$ vs 10$)$ and the force field used than the absolute composition. Within each force field both compositions yielded similar densities close to the experimental values. The simulated rotational anisotropy decay times for the water were on the same order of magnitude for the $w_{0}$ values in each force field. The simulations of spherically restrained systems were used to provide a "control," with which, for example, we could establish that water dynamics in the restrained RMs are similar to water dynamics in the unrestrained RMs in which the water/surfactant interface is structurally distinct. Shape parameters calculated from the moments of inertia also yielded comparable results indicating rod-like shapes for the CHARMM systems and disc- or donutlike shapes for the GROMOS systems.

There exist substantial differences in the interpretation of reverse micelle structure and dynamics in the literature. ${ }^{6,18,22,24,25}$ Most simulation studies support the conclusion that there are significant shape fluctuations, away from the idealized spherical geometry, that are important to the overall system energetics and dynamics. ${ }^{18,23,47}$ However, many experimental studies are interpreted with the assumption of an idealized spherical geometry of the reverse micelle and internal water pool. ${ }^{5,7,48,49}$ Moreover, simulation studies indicate a broad distribution of relaxation times for water rotational anisotropy, with significant force field dependence. Further experimental studies of both the structure and dynamics of reverse micelle systems are needed to clarify the nature of reverse micelle structure and to evaluate critically the predictions of simulation models.

\section{ASSOCIATED CONTENT}

\section{S Supporting Information}

Further analysis of the GROMOS systems including some comparison between the CHARMM and GROMOS force fields. This material is available free of charge via the Internet at http://pubs.acs.org.

\section{AUTHOR INFORMATION}

\section{Corresponding Author}

*E-mail: straub@bu.edu.

\section{Present Address}

${ }^{\ddagger}$ A.M.: Loras College, Department of Chemistry, Dubuque, IA 52001, United States.

\section{Notes}

The authors declare no competing financial interest.

\section{ACKNOWLEDGMENTS}

The authors gratefully acknowledge the support of a grant from the National Institutes of Health (RO1 GM076688), the resources of the Center for Computational Science at Boston University, the Schlumberger Foundation Faculty for the Future Program and CONACYT. This work used the Extreme Science and Engineering Discovery Environment (XSEDE), which is supported by National Science Foundation Grant No. OCI-1053575.

\section{REFERENCES}

(1) Hoar, T.; Schulman, J. Transparent Water-in-Oil Dispersions: The Oleopathic Hydro-Micelle. Nature 1943, 152, 102-103.

(2) Levinger, N. E. Water in Confinement. Science 2002, 298, 17221723.

(3) Thompson, K. F.; Gierasch, L. M. Conformation of a Peptide Solubilizate in a Reversed Micelle Water Pool. J. Am. Chem. Soc. 1984, $106,3648-3652$.

(4) Mukherjee, S.; Chowdhury, P.; Gai, F. Tuning the Cooperativity of the Helix-Coil Transition by Aqueous Reverse Micelles. J. Phys. Chem. B 2006, 110, 11615-11619.

(5) Mukherjee, S.; Chowdhury, P.; Gai, F. Effect of Dehydration on the Aggregation Kinetics of Two Amyloid Peptides. J. Phys. Chem. B 2009, 113, 531-535.

(6) Martinez, A. V.; DeSensi, S. C.; Dominguez, L.; Rivera, E.; Straub, J. E. Protein Folding in a Reverse Micelle Environment: The Role of Confinement and Dehydration. J. Chem. Phys. 2011, 134, 055107.

(7) De, T. K.; Maitra, A. Solution Behaviour of Aerosol OT in NonPolar Solvents. Adv. Colloid Interface Sci. 1995, 59, 95-193.

(8) Nave, S.; Eastoe, J.; Heenan, R. K.; Steytler, D.; Grillo, I. What Is So Special about Aerosol-OT? 2. Microemulsion Systems. Langmuir 2000, 16, 8741-8748.

(9) Maitra, A. Determination of Size Parameters of Water-Aerosol OT-Oil Reverse Micelles from Their Nuclear Magnetic Resonance Data. J. Phys. Chem. 1984, 88, 5122-5125.

(10) Amararene, A.; Gindre, M.; Huérou, J.-Y. L.; Urbach, W.; Valdez, D.; Waks, M. Adiabatic Compressibility of AOT Sodium Bis2ethylhexylsulfosuccinate Reverse Micelles: Analysis of a Simple Model Based on Micellar Size and Volumetric Measurements. Phys. Rev. E 2000, 61, 682-689.

(11) Eicke, H.-F.; Rehak, J. On the Formation of Water/OilMicroemulsions. Helv. Chem. Acta 1976, 59, 2883-2891.

(12) Law, S. J.; Britton, M. M. Sizing of Reverse Micelles in Microemulsions using NMR Measurements of Diffusion. Langmuir 2012, 28, 11699-11706.

(13) Hauser, H.; Haering, G.; Pande, A.; Luisi, P. Interaction of Water with Sodium Bis(2-ethyl-1-hexyl) Sulfosuccinate in Reversed Micelles). J. Phys. Chem. 1989, 93, 7869-7876.

(14) Levinger, N. E.; Swafford, L. A. Ultrafast Dynamics in Reverse Micelles. Annu. Rev. Phys. Chem. 2009, 60, 385-406.

(15) Faeder, J.; Ladanyi, B. M. Molecular Dynamics Simulations of the Interior of Aqueous Reverse Micelles. J. Phys. Chem. B 2000, 104, 1033-1046.

(16) Faeder, J.; Ladanyi, B. M. Solvations Dynamics in Aqueous Reverse Micelles: A Computer Simulations Study. J. Phys. Chem. B 2001, 105, 11148-11158.

(17) Faeder, J.; Ladayni, B. M. Solvation Dynamics in Reverse Micelles: The Role of Headgroup-Solute Interactions. J. Phys. Chem. B 2005, 109, 6732-6740.

(18) Abel, S.; Sterpone, F.; Bandyopadhyay, S.; Marchi, M. Molecular Modeling and Simulations of AOT-Water Reverse Micelles in Isooctane: Structural and Dynamic Properties. J. Phys. Chem. B 2004, 108, 19458-19466. 
(19) Mudzhikova, G.; Brodskaya, E. Molecular Simulation of an Aerosol OT Reverse Micelle: 1 . The Shape and Structure of a Micelle. Colloid J. 2006, 68, 729-737.

(20) Brodskaya, E. N.; Mudzhikova, G. V. Molecular Dynamics Simulation of AOT Reverse Micelles. Mol. Phys. 2006, 104, 36353645.

(21) Mudzhikova, G.; Brodskaya, E. Molecular Simulation of an Aersol OT Reverse Micelle: 2. Energy and Kinetic Characteristics. Colloid J. 2006, 68, 738-742.

(22) Chowdhary, J.; Ladanyi, B. M. Molecular Dynamics Simulation of Aerosol-OT Reverse Micelles. J. Phys. Chem. B 2009, 113, 1502915039 .

(23) Tian, J.; Garcia, A. E. Simulations of the Confinement of Ubiquitin in Self-Assembled Reverse Micelles. J. Chem. Phys. 2011, 124, 225101

(24) Gardner, A.; Vásquez, V.; Clifton, A.; Graeve, O. Molecular Dynamics Analysis of the AOT/Water/Isooctane System: Effect of Simulation Time, Initial Configuration, and Model Salts. Fluid Phase Equilib. 2007, 262, 264-270.

(25) Vasquez, V. R; Williams, B. C.; Graeve, O. A. Stability and Comparative Analysis of AOT/Water/Isooctane Reverse Micelle System Using Dynamic Light Scattering and Molecular Dynamics. J. Phys. Chem. B 2011, 115, 2979-2987.

(26) MacKerell, A. D.; Bashford, D.; Bellott, M., Jr.; R. L, D.; Evanseck, J. D.; Field, M. J.; Fischer, S.; Gao, J.; Guo, H.; Ha, S.; Joseph-McCarthy, D.; Kuchnir, L.; Kuczera, K.; Lau, F. T. K.; Mattos, C.; Michnick, S.; Ngo, T.; Nguyen, D. T.; Prodhom, B., III; W. E, R.; Roux, B.; Schlenkrich, M.; Smith, J. C.; Stote, R.; Straub, J. E.; Watanabe, M.; Wiórkiewicz-Kuczera, J.; Yin, D.; Karplus, M. All-Atom Empirical Potential for Molecular Modeling and Dynamics Studies of Proteins. J. Phys. Chem. B 1998, 102, 3586-3616.

(27) Oostenbrink, C.; Villa, A.; Mark, A. E.; van Gunsteren, W. F. A Biomolecular Force Field Based on the Free Enthalpy of Hydration and Solvation: The GROMOS Force-Field Parameter Sets 53A5 and 53A6. J. Comput. Chem. 2004, 25, 1656-1676.

(28) Phillips, J. C.; Braun, R.; Wang, W.; Gumbart, J.; Tajkhorshid, E.; Villa, E.; Chipot, C.; Skeel, R. D.; Kaleé, L.; Schulten, K. Scalable Molecular Dynamics with NAMD. J. Comput. Chem. 2005, 26, 17811802.

(29) Martyna, G. J.; Tobias, D. J.; Klein, M. L. Constant Pressure Molecular Dynamics Algorithms. J. Chem. Phys. 1994, 101, 41774189 .

(30) Feller, S. E.; Zhang, Y.; Pastor, R. W.; Brooks, B. R. Constant Pressure Molecular Dynamics Simulation: The Langevin Piston Method. J. Phys. Chem. 1995, 103, 4613-4621.

(31) Berendsen, H.; Postma, J.; van Gunsteren, W.; DiNola, A.; Haak, J. Molecular Dynamics with Coupling to an External Bath. J. Chem. Phys. 1984, 81, 3684-3690.

(32) Michaud-Agrawal, N.; Denning, E. J.; Woolf, T. B.; Beckstein, O. Software News and Updates MDAnalysis: A Toolkit for the Analysis of Molecular Dynamics Simulations. J. Comput. Chem. 2011, 32, 2319-2327.

(33) Impey, R.; Madden, P.; McDonald, I. Spectroscopic and Transport Properties of Water. Mol. Phys. 1982, 46, 513-539.

(34) Ghosh, S. A Model for the Orientational Order in Liquid Crystals. Il Nuovo Cimento 1984, 4, 229-244.

(35) Pieniazek, P. A.; Lin, Y.-S.; Chowdhary, J.; Ladanyi, B. M.; Skinner, J. L. Vibrational Spectroscopy and Dynamics of Water Confined inside Reverse Micelles. J. Phys. Chem. B 2009, 113, 1501715028 .

(36) Piletic, I. R.; Moilanen, D. E.; Spry, D.; Levinger, N. E.; Fayer, M. Testing the Core/Shell Model of Nanoconfined Water in Reverse Micelles Using Linear and Nonlinear IR Spectroscopy. J. Phys. Chem. A 2006, 110, 4985-4999.

(37) Fayer, M. D. Dynamics of Water Interacting with Interfaces, Molecules, and Ions. Acc. Chem. Res. 2012, 45, 3-14.

(38) Biswas, R.; Chakraborti, T.; Bagchi, B.; Ayappa, K. NonMonotonic, Distance Dependent Relaxation of Water in Reverse
Micelles: Propagation of Surface Induced Frustration Along Hydrogen Bond Networks. J. Chem. Phys. 2012, 137, 014515.

(39) Laage, D.; Thompson, W. H. Reorientation Dynamics of Nanoconfined Water: Power-Law Decay Hydrogen-Bond Jumps, and a Test of a Two-State Model. J. Chem. Phys. 2012, 136, 044513.

(40) Marchi, M.; Sterpone, F.; Ceccarelli, M. Water Rotational Relaxation and Diffusion in Hydrated Lysozyme. J. Am. Chem. Soc. 2002, 124, 6786-6791.

(41) Pizzitutti, F.; Marchi, M.; Sterpone, F.; Rossky, P. J. How Protein Surfaces Induce Anomalous Dynamics of Hydration Water. J. Phys. Chem. B 2007, 111, 7584-7590.

(42) Lu, W.; Kim, J.; Qiu, W.; Zhong, D. Femtosecond Studies of Tryptophan Solvation: Correlation Function and Water Dynamics at Lipid Surfaces. Chem. Phys. Lett. 2004, 388, 120-126.

(43) Johnston, D. Stretched Exponential Relaxation Arising from a Continuous Sum of Exponential Decays. Phys. Rev. B 2006, 74, 184430 .

(44) Scodinu, A.; Fourkas, J. T. Comparison of the Orientational Dynamics of Water Confined in Hydrophobic and Hydrophili Nanopors. J. Phys. Chem. B 2002, 106, 10292-10295.

(45) Farrer, R. A.; Fourkas, J. T. Orientational Dynamics if Liquids Confined in Nanoporous Sol-Gel Glasses Studied by Optical Kerr Effect Spectroscopy. Acc. Chem. Res. 2003, 36, 605-612.

(46) Pastor, R. W.; A. D. MacKerell, J. Development of the CHARMM Force Field of Lipids. J. Phys. Chem. Lett. 2011, 2, 15261532.

(47) Tian, J.; Garcia, A. E. An Alpha-Helical Peptide in AOT Micelles Prefers to be Localized at the Water/Headgroup Interface. Biophys. J. 2009, 96, 57-59.

(48) Kotlarchyk, M.; Huang, J. S. Structure of Reversed Micelles Determined by Small-Angle Neutron Scattering. J. Phys. Chem. 1985, $89,4382-4386$

(49) Mukherjee, S.; Chowdhury, P.; Gai, F. Infrared Study of the Effect of Hydation on the Amide I Band and Aggregation Properties of Helical Peptides. J. Phys. Chem. B 2007, 111, 4596-4602. 